\title{
FLORÍSTICA E CARACTERIZAÇÃO DE UMA ÁREA DE CAMPO FERRUGINOSO NO COMPLEXO MINERÁRIO ALEGRIA, SERRA DE ANTÔNIO PEREIRA, OURO PRETO, MINAS GERAIS, BRASIL ${ }^{1}$
}

\author{
Eduardo Silva Ataíde ${ }^{2}$, Paulo de Tarso Amorim Castro ${ }^{3}$ e Geraldo Wilson Fernandes ${ }^{4}$
}

\begin{abstract}
RESUMO - Este estudo apresenta o inventário florístico de uma área de campo ferruginoso localizada na Serra de Antônio Pereira, na região Sul-Sudeste do Quadrilátero Ferrífero, no município de Ouro Preto, Minas Gerais. Para a coleta de dados foram demarcadas 15 unidades amostrais de 10 x 10 m em cada tipologia física de substrato estudado: a canga couraçada e o afloramento de Itabirito. Todas as unidades amostrais ocupavam um mesmo perfil de inclinação com azimute NE. As campanhas de campo estenderam-se de março de 2007 a outubro de 2008 e foram realizadas, em média, semanalmente. Nas coletas, restringiram-se apenas as espécies fanerógamas encontradas dentro das unidades amostrais e nas áreas de entorno. Foram inventariadas 182 espécies reunidas em 47 famílias, sendo 102 espécies dentro das unidades amostrais e 80 nas áreas de entorno. As famílias com maior riqueza de espécies nas unidades amostrais foram: Asteraceae (14 spp), Poaceae e Orchidaceae (8 spp cada), Fabaceae e Melastomataceae (7 spp cada), Myrtaceae e Verbenaceae (6 spp cada) e Cyperaceae, Lauraceae, Rubiaceae e Euphorbiaceae (4 spp cada). Os ambientes estudados - canga couraçada e afloramento de Itabirito - apresentaram-se similares.
\end{abstract}

Palavras-chave: Unidades amostrais, Serra de Antônio Pereira e Canga couraçada.

\section{FLORISTIC AND CHARACTERIZATION OF THE RUPESTRIAN FERRUGINOUS FIELD AREA IN THE ALEGRIA COMPLEX MINING, SERRA DE ANTÔNIO PEREIRA, OURO PRETO, MINAS GERAIS, BRAZIL}

\begin{abstract}
This study presents the floristic inventory of the rupestrian ferruginous field areas located inSerra de Antônio Pereira, in the south-southeast of Quadrilátero Ferrífero region in Ouro Preto, MG. There, two different substratcts were surveyed: the canga couraçada, a hard ferruginous laterite, and itabirite rock outcrop. In each substract, 15 sample units $(10 \mathrm{mx} 10 \mathrm{~m})$ were studied. All the samples units were made with the same slope. The data collected were only restricted to the phanerogam species found in the samples units and surrounding areas. The floristic inventory done at Serra de Antônio Pereira found 182 species united in 47 families, of which 102 species occur inside the sample units and other 80 species in surrounding areas. The richest species families in the samples units were: Asteraceae (14 spp), Poaceae and Orchidaceae (8 spp each), Fabaceae and Melastomataceae (7 spp each), Myrtaceae and Verbenaceae (6 spp each), Cyperaceae, Lauraceae, Rubiaceae and Euphorbiaceae (4 spp each). Both the studied units, canga couraçada and itabirite outcrop, showed similarities.
\end{abstract}

Keywords: Sample units, Serra de Antônio Pereira and Canga couraçada.

\footnotetext{
${ }^{1}$ Recebido em 07.04.2010 e aceito para publicação em 31.10.2011.

${ }^{2}$ Faculdade Presidente Antonio Carlos de Congonhas, UNIPAC de Congonhas/MG. E-mail: <eduardosataide@yahoo.com.br>.

${ }^{3}$ Departamento de Geologia da Universidade Federal de Ouro Preto, DEGEO/UFOP. E-mail: <paulo_de_castro@degeo.ufop.br>.

${ }^{4}$ Instituto de Ciências Biológicas da UFMG. E-mail: <gw.fernandes@gmail.com>.
} 


\section{INTRODUÇÃO}

A Serra do Espinhaço é uma cadeia montanhosa que se estende de norte a sul nos Estados da Bahia e de Minas Gerais, em altitudes que variam de 700 a 2.000 m (HARLEY, 1995). Nesses ambientes predominam um tipo vegetacional denominado campos rupestres, que podem estar associados a afloramentos de rochas quartzíticas, graníticas ou ferruginosas. Campos rupestres são aqueles que se desenvolvem sobre afloramentos rochosos ou sobre solos primários com diferentes graus de desagregação da rocha, podendo diferir quanto à composição florística de acordo com a natureza geológica do substrato.

O termo campo rupestre foi utilizado por Magalhães (1966) para designar o tipo de vegetação associada a afloramentos quartzíticos na Serra do Espinhaço (VIANA; LOMBARDI, 2007). Uma vez que o termo campo rupestre vem sendo utilizado particularmente para a vegetação associada a substratos quartzíticos (MAGALHÃES, 1966; GIULIETTI et al., 1987), é recomendável que se discrimine o tipo de substrato ao se tratar de outros campos rupestres, como o campo rupestre sobre substrato granítico e o campo rupestre hematítico ou sobre rocha ferruginosa (VINCENT, 2004).

O termo Campo Ferruginoso foi primeiramente usado por Rizzini (1979) para classificar esse tipo de vegetação que se desenvolve sobre substrato rico em ferro e vem sendo utilizado atualmente por pesquisadores como Vincent (2004) e Vincent et al. (2002).

Essa fitofisionomia, comum no Quadrilátero Ferrífero, em Minas Gerais e na Serra dos Carajás, Pará, é caracterizada por vegetação herbáceo-arbustiva, que possui diversas adaptações para seu estabelecimento nesse ambiente adverso (VINCENT, 2004). Entre essas adaptações está a capacidade de acúmulo de metais pesados em tecidos vegetais, como mostram estudos realizados na Serra dos Carajás por Porto e Silva (1989) e Silva (992) e no Quadrilátero Ferrífero por Teixeira e Lemos-Filho (1998).

Os trabalhos sobre inventários florísticos em campos ferruginosos ainda são escassos, dos quais se podem destacar os trabalhos de Secco e Mesquita (1983), Silva e Rosa (1990) e Silva et al. (1996) e Cleef e Silva (1994), estes realizados na região de Carajás, Pará. Em relação ao Quadrilátero Ferrífero, destacam-se os trabalhos de Brandão e Gavilanes (1990), Roschel (2000), Vincent (2004), Viana e Lombardi (2007), Mourão e Stehmann (2007) e Jacobi et al. (2008).
O Quadrilátero Ferrífero ocupa uma área de 7.000 km² $^{2}$ DORR, 1969), e situa-se na zona limítrofe entre dois hotspots brasileiros: Mata Atlântica e Cerrado. Localiza-se adjacente ao limite austral da Cadeia do Espinhaço, e sua flora distribui-se em diversas formações vegetais, caracterizadas por um mosaico de influências biogeográficas que evidencia o caráter ecotonal da região. São encontradas na região do Quadrilátero Ferrífero formações florestais (Floresta Estacional Semidecidual e Floresta Ombrófila), savânicas (Cerrado sensu stricto, campos sujos e campos limpos) e rupestres (Campo Rupestre sobre Quartzito, Campo Rupestre sobre Canga e, mais raramente, Campos sobre Afloramento Granítico). A região conta com extrema diversidade florística, incluindo diversas espécies endêmicas, ameaçadas de extinção e de uso potencial como medicinais, ornamentais, alimentícias ou madeireiras (VIANA, 2008).

O Quadrilátero Ferrífero é considerado uma região de maior diversidade florística daAmérica do Sul (HARLEY, 1995; GIULIETTI et al., 1997 apud JACOBI; CARMO, 2008), com mais de $30 \%$ de endemismo em sua flora (GIULIETTI et al., 1987 apud JACOBI; CARMO, 2008), prioritária para a conservação da biodiversidade no Estado de Minas Gerais, por possuir os campos ferruginosos com ocorrência de espécies vegetais restritas à região e por constituir um ambiente único em Minas (JACOBI; CARMO, 2008). Essas áreas se encontram ameaçadas pela expansão das minerações, atividade que explora a hematita ocorrente sob os itabiritos.

A grande diversidade geológica e topográfica da região, de fato, é um dos motivos para a existência de riquíssima biodiversidade, o que justificou o reconhecimento do Quadrilátero Ferrífero como uma região de “importância biológica extrema” (DRUMMOND et al., 2005) e sua inserção dentro da Reserva da Biosfera da Cadeia do Espinhaço pela UNESCO em 2005 (JACOBI, 2008).

O objetivo deste trabalho foi contribuir para o conhecimento da flora fanerogâmica de uma área de campo ferruginoso com vistas a subsidiar programas de restauração de áreas futuramente degradadas pela mineração.

\section{MATERIAL E MÉTODOS}

A Serra de Antônio Pereira abrange parte do Sinclinal Mariana localizado na região Sul-Sudeste do Quadrilátero Ferrífero e está inserida, de forma disjunta, no Complexo da Cadeia do Espinhaço. 
O clima dominante da região é do tipo Cwa de Köeppen, temperado quente, com estação seca de abril a setembro. A temperatura média anual é de $20^{\circ} \mathrm{C}$, e a precipitação média anual varia de 1.300 mm, na porção Leste do Quadrilátero, a 2.100 mm, na porção Sul, em Ouro Preto (HERZ, 1978).

A área de estudo localiza-se no Complexo Minerário denominado Alegria 7, de propriedade da Samarco Mineração S.A., possui área de 225,6 ha e encontra-se no extremo Norte do município de Ouro Preto, divisa com o município de Mariana. As unidades amostrais foram plotadas a partir do ponto 0655333 e UTM 7768414 (Datum SAD 69, fuso 23 S).

Foram distribuídas 15 unidades amostrais de 10 x 10 m, todas no mesmo perfil de declividade e com azimute NE, em cada tipologia física de substrato estudado: a canga couraçada sensu (RIZZINI, 1979) e o afloramento de itabirito, perfazendo um total de 30 unidades. As áreas de canga ocorrem nas meias-vertentes, enquanto os afloramentos predominam nas quotas mais altas do perfil, que variam de $900 \mathrm{~m}$ a $1.200 \mathrm{~m}$.

O inventário florístico foi realizado entre março de 2007 e outubro de 2008, com incursões semanais, em média, no campo. As coletas foram restritas às espécies fanerógamas em estado fértil, abrangendo as unidades amostrais e a área de entorno. As espécies estéreis foram marcadas para posteriores coleta e identificação. Todo o material coletado encontra-se depositado no Herbário José Badini (OUPR), da Universidade Federal de Ouro Preto, em Ouro preto, MG.

A identificação das espécies foi por meio de bibliografia especializada, comparação com exsicatas determinadas depositadas no Herbário José Badini (OUPR) e por colaboração de especialistas de outras instituições. A circunscrição das famílias adotada foi aquela proposta pelo APG II (APG, 2003).

A metodologia utilizada para a amostragem fitossociológica foi o método de Relevè (MUELLERDOMBOIS; ELLENBERG, 1974), a qual consistiu em demarcar Relevès (unidades amostrais) de 10 x 10 m em cada tipo de substrato estudado. Os parâmetros fitossociológicos foram coletados nas 30 unidades amostrais utilizadas no inventário florístico.

Para determinar a frequência das espécies, foram estimados dois parâmetros: a Frequência Absoluta (Fai), que é a probabilidade de se encontrar pelo menos um indivíduo da espécie i em uma unidade amostral; e a
Frequência Relativa (FRi), que equivale à razão entre a frequência absoluta de determinada espécie e a somatória das frequências absolutas de todas as espécies amostradas. Também, foi calculado o Índice Valor de Importância (IVI), que é a soma dos valores de Frequência Relativa (FRi) e Dominância Relativa (DoRi).

\section{RESULTADOS}

O inventário florístico das fanerógamas contou com 182 espécies reunidas em 47 famílias, das quais 102 espécies foram inventariadas nas unidades amostrais (Tabela 1) e 80 nas áreas de entorno (Tabela 2). As 102 espécies inventariadas nas unidades amostrais estão reunidas em 34 famílias, abrangendo 81 gêneros, sendo 73 espécies $(71,56 \%)$ de dicotiledôneas e 29 (28,43\%) de monocotiledôneas. As famílias com maior riqueza são: Asteraceae (14 spp), Orchidaceae e Poaceae (8 spp cada), Melastomataceae e Fabaceae (7 spp cada), Myrtaceae e Verbenaceae (6 spp cada) e Rubiaceae, Lauraceae, Cyperaceae e Euphorbiaceae (4 spp cada).

Dos hábitos de crescimento ocorrentes na área de estudo, 37\% eram constituídos por espécies herbáceas, $51 \%$ de arbustos, $8 \%$ de subarbustos e $4 \%$ de lianas.

Nos ambientes estudados canga couraçada e afloramento de itabirito, as espécies apresentavam-se bem distribuídas, sendo os ambientes bem similares ( $\mathrm{H}^{\prime}$ canga $=3,26$ nats/ind e $\mathrm{H}^{\prime}$ afloramento $=3$,20 nats/ ind). Entretanto, 34 espécies foram inventariadas exclusivamente em canga: Achyrocline satureioides, Aegiphylla sp, Axonopus siccus, Blepharocalix salicifolius, Calyptranthes pteropoda, Cambessedesia pitirofila, Cinnamomum sellowianum, Declieuxia fruticosa, Dicronema sp1, Dioscorea filiformes, Epidendrum secundum, Lantana trifolia, Miconia coralina, Miconia theaezans, Microlicia crenulata, Microtea tenuiflora, Myrcia eriocalix, Myrcia splendens, Nectandra nitidula, Ouratea semiserrata, Paepalanthus hilairei, Panicum wettsteinii, Pera obtusifolia, Phyllanthus klotzchianus, Phylodendron sp, Remijia ferruginea, Schefflera morotononi, Sebastiania corniculata, Stirax comporum, Stylosanthes ruelioides, Tibouchina alba, Vellozia graminea, Vernonia holosericea e Vochysia emarginata. Outras nove espécies foram inventariadas exclusivamente nos afloramentos de Itabirito, como Ditassa linearis, Dasyphyllum sprengelyanum, Eremanthus incanus, Hyptis monticula, Hololepis pedunculata, Mandevilla tenuiflora, Matayba marginata, Nematanthus strigillosus e Trichogonia sp. As demais (59 spp) apresentavam-se de forma generalista e ocorriam nos dois ambientes. 
Tabela 1 - Espécies de plantas fanerógamas inventariadas nas unidades amostrais nos campos ferruginosos da Alegria 7 - Samarco Mineração S.A., com seus ambientes de ocorrência (canga couraçada = Cc e afloramento de Itabirito = IT), Número de Herbário (NH) e Formas de Vida (FV). Fan = Fanerófita, Geo = Geófita, Hem = Hemicriptófita, Cam $=$ Caméfita e Ter $=$ Terófita .

Table 1 - Species of phanerogam plants inventoried in the samples units in rupestrian ferruginous field of Alegria 7 - Samarco Mineração S.A., with occurrence sites (Canga couraçada = Cc and Itabirite outcrops = IT), herbarium number $(\mathrm{NH})$ and life forms $(\mathrm{FV})$, Geo $=$ Geophytes, Hem = Hemicryptophytes, Cam = Chamaephytes and Ter $=$ Therophytes.

\begin{tabular}{l}
\hline Família \\
\hline Apocynaceae \\
Araceae \\
Araliaceae \\
Aristolochiaceae \\
Asteraceae
\end{tabular}

\section{Bromeliaceae \\ Cyperaceae \\ Dioscoreaceae \\ Ericaceae \\ Eriocaulaceae \\ Euphorbiaceae}

Fabaceae

Gesneriaceae

Iridaceae

Lamiaceae

Lauraceae

Espécie

Ditassa linearis Mart.

Ditassa mucronata Mart.

Mandevilla tenuiflora (J.C. Mikan) Woodson

Anthurium minarum Sakur. \& Mayo Philodendron $s p$

Schefflera morototoni (Aubl.) Maquire et al. Aristolochia saxicola Hoene

Achyrocline satureioides (Lam.) DC. Baccharis punctulata DC.

Baccharis reticularia DC.

Dasyphyllum sprengelianum (Gardner) Cabrera

Eremanthus erythropappus (DC.) MacLeish

Eremanthus incanus (Less.) Less. Eupatorium $s p$

Eupatorium squalidum DC.

Hololepis pedunculata (DC. ex Pers.) DC. Lychnophora pinaster Mart.

Lychnophora syncephala (Sch. Bip.) Sch. Bip. Trichogonia sp

Eremanthus crotonoides (DC.) Sch.Bip.

Vernonia holocericea Mart. ex DC.

Cryptanthus schwackeanus Mez

Dyckia rariflora Schult. \& Schult.f.

Bulbostylis glaziovii (Boeckeler) C.B. Clarcke Dicronema sp1

Fintelmannia microstachya (Nees ex Arn) H. Pfeif Lagenocarpus rigidus Nees

Dioscorea filiformis Griseb.

Leucothoe cordifolia Meisn.

Paepalanthus hilairei Körn Croton lobatus L.

Croton oleoides Müll. Arg.

Pera obtusifolia (Schott) Müll. Arg.

Sebastiania corniculata Müll. Arg

Aeschynomene elegans Schltdl. \& Cham.

Bauhinia rufa (Bong.) Steud.

Centrosema coriaceum Benth. Galactia martii DC.

Periandra mediterranea (Vell.) Taub. Senna reniformis (G.Don) H.S.Irwin \& Barneby Stylosanthes ruellioides Mart.

Nematanthus strigillosus (Mart.) H.E.Moore Paliavana sericifolia Benth. Neomarica $s p$

Hyptis monticula Mart. ex Benth.

Cinnamomum erythropus (Nees \& Mart.) Kosterm. Cinnamomum sellowianum (Nees \& Mart.) Kosterm.

Ambiente

FV

NH

\begin{tabular}{|c|c|c|}
\hline IT & Cam & OUPR 20618 \\
\hline IT/Cc & Cam & OUPR 21127 \\
\hline I T & Ter & OUPR 20731 \\
\hline IT/Cc & Geo & OUPR 20538 \\
\hline Cc & Geo & OUPR 20770 \\
\hline Сс & Fan & OUPR 21017 \\
\hline $\mathrm{IT} / \mathrm{Cc}$ & Hem & OUPR 21319 \\
\hline Cc & Fan & OUPR 20726 \\
\hline IT/Cc & Fan & OUPR 21302 \\
\hline IT/Cc & Fan & OUPR 20721 \\
\hline IT & Fan & OUPR 20722 \\
\hline IT/Cc & Fan & OUPR 20728 \\
\hline IT & Fan & OUPR 20733 \\
\hline IT/Cc & Fan & OUPR 21301 \\
\hline IT/Cc & Fan & OUPR 19436 \\
\hline IT & Fan & OUPR 19417 \\
\hline IT/Cc & Fan & OUPR 19428 \\
\hline IT/Cc & Fan & OUPR 20600 \\
\hline I T & Fan & OUPR 19445 \\
\hline IT/Cc & Fan & OUPR 20727 \\
\hline Cc & Fan & OUPR 20624 \\
\hline IT/Cc & Hem & OUPR 21718 \\
\hline IT/Cc & Hem & OUPR 19418 \\
\hline $\mathrm{IT} / \mathrm{Cc}$ & Hem & OUPR 21304 \\
\hline Cc & Hem & OUPR 21010 \\
\hline IT/Cc & Hem & OUPR 20709 \\
\hline IT/Cc & Hem & OUPR 20548 \\
\hline Сc & Hem & OUPR 21297 \\
\hline IT/Cc & Fan & OUPR 21200 \\
\hline Cc & Fan & OUPR 19440 \\
\hline $\mathrm{IT} / \mathrm{Cc}$ & Fan & OUPR 19420 \\
\hline IT/Cc & Fan & OUPR 19438 \\
\hline Cc & Fan & OUPR 21644 \\
\hline Cc & Fan & OUPR 20837 \\
\hline $\mathrm{IT} / \mathrm{Cc}$ & Ter & OUPR 21238 \\
\hline IT/Cc & Fan & OUPR 21239 \\
\hline IT/Cc & Cam & OUPR 20719 \\
\hline IT/Cc & Cam & OUPR 20916 \\
\hline $\mathrm{IT} / \mathrm{Cc}$ & Fan & OUPR 19435 \\
\hline IT/Cc & Fan & OUPR 20725 \\
\hline Cc & Cam & OUPR 19448 \\
\hline IT & Cam & OUPR 19449 \\
\hline IT/Cc & Fan & OUPR 19439 \\
\hline IT/Cc & Geo & OUPR 20850 \\
\hline I T & Fan & OUPR 20917 \\
\hline IT/Cc & Fan & OUPR 20542 \\
\hline Cc & Fan & OUPR 20688 \\
\hline
\end{tabular}

Revista Árvore, Viçosa-MG, v.35, n.6, p.1265-1275, 2011 
Tabela 1 - Cont.

Table 1 - Cont

$\overline{\text { Nectandra nitidula }} \overline{\mathrm{Ness}}$

Ocotea tristis (Nees \& Mart.) Mez

Lythraceae

Malpighiaceae

Melastomataceae

Myrsinaceae

Myrtaceae

Ochnaceae

Orchidaceae

Passifloraceae

Phyllanthaceae

Phytolacaceae

Poaceae

Polygonaceae

Rubiaceae

Sapindaceae Styracaceae

Velloziaceae
Diplusodon buxifolius (Cham. \& Schltdl.) A.DC.

Byrsonima variabilis A.Juss.

Heteropterys campestris A.Juss.

Cambessedesia pityrophylla (Mart. ex DC.)

$$
\text { A.B.Martins }
$$

Leandra atropurpurea Cogn.

Miconia corallina Spring

Miconia theaezans (Bonpl.) Cogn.

Microlicia crenulata (DC) Mart. Tibouchina alba Cogn.

Tibouchina multiflora (Gardner) Cogn. Myrcine lancifolia Mart.

Blepharocalyx salicifolius (Kunth) O.Berg

Calyptranthes pteropoda O.Berg

Myrcia pulchra (O.Berg) Kiaersk.

Myrcia splendens (Sw.) DC.

Myrcia eriocalix DC.

Myrcia venulosa DC.

Ouratea semiserrata (Mart. \& Nees) Engl.

Acianthera teres (Lindl.) Borba

Epidendrum martianum Lindl.

Epidendrum secundum Jacq.

Hoffmannseggella crispata (Thunb.) H.G.Jones

Hoffmannseggella cinnabarina (Batem. ex Lindl.)H.G.Jones

Oncidium gracile Lindl.

Oncidium blanchetii Rchb. f.

Sophronitis caulescens (Lindl.) Van den

Berg \& M.W. Chase

Passiflora villosa Vell.

Phyllanthus klotzschianus Müll.Arg. Microtea tenuiflora Moq.

Apochloa poliophylla (Renvoize \& Zuloaga) Zuloaga \& Morrone

Axonopus laxiflorus (Trin.) Chase

Axonopus siccus (Nees) Kuhlm.

Ichnanthus bambusiflorus (Trin.) Döll

Panicum pseudisachne Mez

Panicum wettsteinii Hack.

Schizachyrium sanguineum (Retz.) Alston

Schizachyrium tenerum Nees

Coccoloba acrostichoides Cham.

Alibertia rotunda (Cham.) K. Schum.

Declieuxia fruticosa (Willd. ex Roem. \& Schult.) Kuntze

Psyllocarpus laricoides Mart. ex Mart. \& Zucc.

Remijia ferruginea (A.St.-Hil.) DC.

Matayba marginata Radlk.

Styrax camporum Pohl

Vellozia compacta Mart. ex Schult. \& Schult.f. Vellozia graminea Pohl
$\overline{\mathrm{Cc}}-$

Fan

OUPR $216 \overline{58}$

IT/Cc Fan

OUPR 21645

IT/Cc Fan OUPR 19434

IT/Cc Fan OUPR 20599

IT/Cc fan OUPR 20716

Cc Fan OUPR 21015

IT/Cc Fan OUPR 19413

Cc Fan OUPR 21639

Cc Fan OUPR 21640

Cc Fan OUPR 20595

Cc Fan OUPR 21316

IT/Cc Fan OUPR 19427

IT/Cc Fan OUPR 1570

Cc Fan OUPR 20825

Cc Fan OUPR 21685

IT/Cc Fan OUPR 21857

Cc Fan OUPR 20996

Cc Fan OUPR 21289

IT/Cc Fan OUPR 20723

Cc Fan OUPR 20592

IT/Cc Hem OUPR 20544

IT/Cc Hem OUPR 20650

Cc Hem $\quad$ OUPR 21651

IT/Cc Hem OUPR 19412

IT/Cc Hem OUPR 21657

IT/Cc Hem $\quad$ OUPR 21318

IT/Cc Hem OUPR 21655

IT/Cc Hem OUPR 21067

IT/Cc Cam OUPR 21030

Cc $\quad$ Cam OUPR 20594

Cc $\quad$ Ter OUPR 21299

IT/Cc Hem OUPR 21087

IT/Cc Hem $\quad$ OUPR 21086

Cc Hem OUPR 20839

IT/Cc Hem OUPR 20819

IT/Cc Hem OUPR 20834

Cc Hem $\quad$ OUPR 20831

IT/Cc Hem OUPR 21084

IT/Cc Hem OUPR 21077

IT/Cc Fan OUPR 21293

IT/Cc Fan OUPR 21240

$\begin{array}{lll}\text { Cc } & \text { Fan } & \text { OUPR } 19426\end{array}$

IT/Cc Cam OUPR 19456

Cc Fan OUPR 21642

IT Fan OUPR 21036

Cc Fan OUPR 21295

IT/Cc Fan OUPR 21294

Cc Hem OUPR 19454

Continua

Continued... 
Tabela 1 - Cont.

Table 1 - Cont.

\begin{tabular}{|c|c|c|c|c|}
\hline \multirow{6}{*}{ Verbenaceae } & Aegiphylla sp & Сc & Fan & OUPR 20853 \\
\hline & Lantana fucata Lindl. & IT/Cc & Fan & OUPR 21315 \\
\hline & Lantana trifolia L. & Cc & Fan & OUPR 20876 \\
\hline & Lippia glandulosa Schauer & IT/Cc & Fan & OUPR 20720 \\
\hline & Lippia hermannioides Cham. & IT/Cc & Fan & OUPR 19458 \\
\hline & Stachytarpheta glabra Cham. & IT/Cc & Fan & OUPR 19431 \\
\hline Vochysiaceae & Vochysia emarginata (Vahl) Poir. & Сс & Fan & OUPR 20912 \\
\hline
\end{tabular}

Tabela 2 - Inventário florístico fanerogâmico das áreas de entorno das unidades amostrais. NH = Número de Herbário; FV = Formas de Vida; Fan = Fanerófita; Cam = Caméfita; Geo = Geófita; Ter = Terófita; e Hem = Hemicriptófita Table 2 - Species of phanerogam plants inventoried in the around areas of the samples units. Herbarium number (NH); life forms (FV); Geo = Geophytes, Hem = Hemicryptophytes; Cam = Chamaephytes; and Ter = Therophytes.

\begin{tabular}{|c|c|c|c|}
\hline Família & Espécie & FV & NH \\
\hline Acanthaceae & Ruellia macrantha (Mart. ex Ness) Lindau & Fan & OUPR 19460 \\
\hline \multirow[t]{4}{*}{ Apocynaceae } & Ditassa decussata Mart. & Fan & OUPR 20602 \\
\hline & Ditassa laevis Mart. & Fan & OUPR 19424 \\
\hline & Ditassa retusa Mart. & Fan & OUPR 20546 \\
\hline & Tassadia sp & Fan & OUPR 19419 \\
\hline \multirow[t]{3}{*}{ Aquifoliaceae } & Ilex conocarpa Reissek & Fan & OUPR 20918 \\
\hline & Ilex pseudobuxus Reissek & Fan & OUPR 20878 \\
\hline & Ilex sp & Fan & OUPR 20598 \\
\hline Arecaceae & Syagrus sp & Fan & OUPR 19422 \\
\hline \multirow[t]{3}{*}{ Aristolochiaceae } & Aristolochia c.f gracilis Duch. & Cam & OUPR 20763 \\
\hline & Aristolochia c.f smilacina (Klotzsch) Duch. & Cam & OUPR 20717 \\
\hline & Aristolochia fimbriata Cham. & Cam & OUPR 20610 \\
\hline \multirow[t]{13}{*}{ Asteraceae } & Baccharis platypoda DC. & Fan & OUPR 21892 \\
\hline & Baccharis sp & Fan & OUPR 20725 \\
\hline & Calea clematidea Baker & Fan & OUPR 19447 \\
\hline & Eupatorium adamantinum Gardner & Fan & OUPR 20686 \\
\hline & Eupatorium angustissimum Spreng. ex Baker & Fan & OUPR 20944 \\
\hline & Mikania hirsutissima DC. & Cam & OUPR 20549 \\
\hline & Mikania $s p$ & Cam & OUPR 20723 \\
\hline & Richterago amplexifolia (Gardner) Kuntze & Hem & OUPR 20607 \\
\hline & Senecio pohlii Sch.Bip. ex Baker & Fan & OUPR 19443 \\
\hline & Trichogonia hirtiflora (DC.) Sch.Bip. ex Baker & Fan & OUPR 20718 \\
\hline & Trixis divaricata (Kunth) Spreng. & Fan & OUPR 21014 \\
\hline & Vernonia scorpioides (Lam.) Pers. & Fan & OUPR 19457 \\
\hline & Vernonia sp & Fan & OUPR 19415 \\
\hline Begoniaceae & Begonia c.f lobata Schott & Hem & OUPR 22268 \\
\hline Campanulaceae & Siphocampylus sp & Fan & OUPR 19444 \\
\hline \multirow[t]{4}{*}{ Cyperaceae } & Bulbostylis sp & Hem & OUPR 20714 \\
\hline & Cryptangium minarum (Nees) Boeckeler & Hem & OUPR 20914 \\
\hline & Dicronema sp2 & Hem & OUPR 21310 \\
\hline & Rynchospora sp & Hem & OUPR 21129 \\
\hline \multirow[t]{3}{*}{ Convolvulaceae } & Ipomea sp & Cam & OUPR 19441 \\
\hline & Cuscuta racemosa Mart. & Cam & OUPR 20764 \\
\hline & Jaquemontia sp & Cam & OUPR 20615 \\
\hline \multirow[t]{3}{*}{ Ericaceae } & Gaylussacia c.f brasiliensis (Spreng.) Meisn. & Fan & OUPR 20767 \\
\hline & Gaylussacia c.f montana (Pohl) Sleumer & Fan & OUPR 20685 \\
\hline & $\ldots \ldots \ldots$ Leucothoe $s p$ & Fan & OUPR 19425 \\
\hline
\end{tabular}

Revista Árvore, Viçosa-MG, v.35, n.6, p.1265-1275, 2011 
Tabela 2 - Cont

Table 2 - Cont.

\begin{tabular}{|c|c|c|c|}
\hline Eriocaulaceae & Paepalanthus hilairei Körn. & Fan & OUPR 19440 \\
\hline Fabaceae & Chamaecrista mucronata (Spreng.) H.S. Irwin \& Barneby & Fan & OUPR 19416 \\
\hline Gentianaceae & Lisianthus $s p$ & Cam & OUPR 19453 \\
\hline Iridaceae & Sisyrinchium sp & Geo & OUPR 20757 \\
\hline \multirow[t]{2}{*}{ Lamiaceae } & Hyptis homalophylla Pohl ex Benth. & Fan & OUPR 20768 \\
\hline & Hyptis passerina Mart. ex Benth. & Fan & OUPR 20966 \\
\hline Lauraceae & Ocotea diospyrifolia (Meisn.) Mez & Fan & OUPR 21013 \\
\hline Loganiaceae & Spigelia spartiodes Cham. & Fan & OUPR 21296 \\
\hline \multirow[t]{4}{*}{ Malvaceae } & Hibiscus bifurcatus Cav. & Fan & OUPR 19446 \\
\hline & Hibiscus $s p$ & Fan & OUPR 21001 \\
\hline & Pavonia montana Garcke ex Gürke & Fan & OUPR 19452 \\
\hline & Sida spinosa $\mathrm{L}$ & Fan & OUPR 19442 \\
\hline Malpighiaceae & Heteropteris $s p$ & Fan & OUPR 20982 \\
\hline \multirow{4}{*}{ Melastomataceae } & Lavoisiera $s p$ & Fan & OUPR 19451 \\
\hline & Marcetia sp & Fan & OUPR 20597 \\
\hline & Acisanthera variabilis (Mart. \& Schrank) Triana & Fan & OUPR 20631 \\
\hline & Trembleya parviflora (D.Don) Cogn. & Fan & OUPR 21009 \\
\hline \multirow[t]{8}{*}{ Myrtaceae } & Eugenia nutans O. Berg. & Fan & OUPR 20817 \\
\hline & Eugenia racemulosa O. Berg & Fan & OUPR 21001 \\
\hline & Myrcia c.f subavenia (O. Berg) N. Silveira & Fan & OUPR 20802 \\
\hline & Myrcia amazonica DC. & Fan & OUPR 21857 \\
\hline & Myrcia vautheriana O. Berg. & Fan & OUPR 21540 \\
\hline & Myrcia splendens (Sw.) DC. & Fan & OUPR 20996 \\
\hline & Myrcia mutabilis (O. Berg) N. Silveira & Fan & OUPR 20992 \\
\hline & Myrcia detergens Miq. & Fan & OUPR 20801 \\
\hline Ochnaceae & Sauvagesia erecta L. & Cam & OUPR 20696 \\
\hline Oleaceae & Chionanthus filiformis (Vell.) P.S.Green & Fan & OUPR 21928 \\
\hline Orchidaceae & Acianthera ramosa (Barb.Rodr.) F. Barros & Hem & OUPR 21072 \\
\hline \multirow[t]{2}{*}{ Phyllanthaceae } & Phyllanthus rosellus (Müll.Arg.) Müll.Arg. & Cam & OUPR 21317 \\
\hline & Phyllanthus klotzschianus Müll.Arg. & Cam & OUPR 20594 \\
\hline \multirow[t]{4}{*}{ Poaceae } & Andropogon leucostachyus Kunth & Hem & OUPR 20851 \\
\hline & Axonopus pressus (Nees ex Steud.) Parodi & Hem & OUPR 21088 \\
\hline & Andropogon bicornis L. & Hem & OUPR 21557 \\
\hline & Paspalum brachytrichum Hack & Hem & OUPR 20745 \\
\hline Polygalaceae & Polygala oleifolia A.St.-Hil. \& Moq. & Fan & OUPR 20875 \\
\hline \multirow[t]{5}{*}{ Rubiaceae } & Borreria poaya (A.St.-Hil.) DC. & Fan & OUPR 20756 \\
\hline & Borreria sp & Fan & OUPR 19455 \\
\hline & Coccocypselum crassifolium Standl. & Fan & OUPR 20596 \\
\hline & Psychotria pleiocephala Müll. Arg. & Fan & OUPR 20865 \\
\hline & Psychotria sessilis Vell. & Fan & OUPR 20822 \\
\hline Sapindaceae & Paullinia carpopoda Cambess. & Fan & OUPR 20730 \\
\hline Solanaceae & Solanum refractifolium Schltdl. & Fan & OUPR 19430 \\
\hline Velloziaceae & Barbacenia c.f tomentosa Mart. & Hem & OUPR 21314 \\
\hline Vochysiaceae & Vochysia tucanorum Mart. & Fan & OUPR 19459 \\
\hline Verbenaceae & Lippia rubiginosa Schauer & Fan & OUPR 21038 \\
\hline Xyridaceae & Xyris c.f laxifolia Mart. & Hem & OUPR 19450 \\
\hline
\end{tabular}

\section{DISCUSSÃO}

As famílias botânicas com maior diversidade de espécies encontradas neste estudo são também citadas em outros trabalhos realizados em Campos Ferruginosos do Quadrilátero Ferrífero (ROSCHEL, 2000; BRANDÃO et al., 1991, 1994; BRANDÃO et al., 1997; VINCENT, 2004; VIANA; LOMBARDI, 2007). A família Asteraceae, a mais rica em espécies neste estudo, também foi citada em outros trabalhos (MOURÃO; STEHMANN, 2007) como a mais diversa. Silva (1991) citou as famílias Asteraceae, Orchidaceae, Poaceae, Euphorbiaceae, Rubiaceae, Fabaceae, 
Myrtaceae e Cyperaceae como aquelas com ampla distribuição geográfica, sendo consideradas as maiores famílias botânicas do mundo. Também, foram reportadas por Goodland e Ferri (1979) como "super representadas no cerrado”. Aqui, evidentemente, não é correto usar a expressão super representadas, mas são bem representadas por espécies características do tipo de ecossistema em estudo. Deve-se considerar que os trabalhos citados seguiram a circunscrição de Cronquist e este estudo segue a circunscrição proposta pelo APG II (APG, 2003).

Nessa área ocorrem ainda espécies que só foram observadas nas vias de acesso aos ambientes estudados, incluídas neste estudo como áreas de entorno, também inventariadas. Essas vias foram abertas pela empresa e são caracterizadas por apresentarem um substrato friável, constituído por nódulos ferruginosos, semelhantes à canga nodular descrita por Vincent (2004). As espécies ocorrentes exclusivamente nesse ambiente são: Hibiscus bifurcatus, Hibiscus sp, Pavonia montana, Andropogon leucostachyus e Andropogon bicornis. A presença das três primeiras espécies se deve ao fato de elas serem arbustos de médio porte e preferencialmente se estabelecerem nesse ambiente por necessitarem de substrato que lhes permitisse maior penetração de seu sistema radicular. Já as gramíneas A. Bicornis e A. leucostachyus são espécies daninhas ou ruderais (LORENZI, 2000) e possivelmente colonizaram esse ambiente, que foi constituído após a perturbação da canga anteriormente predominante. Segundo Roschel (2000), as espécies da família Eriocaulaceae desenvolvem-se em solos arenosos, onde há acúmulo de água e matéria orgânica. A espécie Paepalanthus hilairei também foi observada com frequência nas áreas perturbadas devido, também, à característica de boa penetrabilidade do sistema radicular naquele substrato.

Nas áreas de meia vertente, onde predominam as cangas, observou-se a ocorrência de pequenos capões formados por arbustos com altura de até $3 \mathrm{~m}$. Nesses sítios foi constatada a presença de uma camada mais espessa de substrato, de natureza coluvionar, permitindo, assim, o desenvolvimento de espécies arbustivas de maior porte, como Aegiphylla sp, Vochysia emarginata, Miconia theaezans, Schefflera morototoni, Eremanthus erythropappus e Syagrus sp. Essas áreas são ecotonais, com as porções de mata ciliar do rio Piracicaba que ocorrem nas partes inferiores do perfil.
Do total de espécies inventariadas neste estudo, sete estão registradas na Lista de Espécies Ameaçadas de Extinção da Fundação Biodiversitas (DRUMMOND et al., 2005). São classificadas como vulneráveis (VU) as seguintes espécies: Ditassa laevis, Calea clematidea, Sophronitis caulescens e Sophronitis crispata. Destacaram-se as espécies Oncidium gracile, Dyckia rariflora e Paspalum brachytrichum, que estão classificadas como criticamente em perigo (CR), sendo a última citada por Viana e Filgueiras (2008) como provavelmente endêmica dos campos ferruginosos do Quadrilátero Ferrífero, com registros apenas nas serras de Capanema e Moeda, além do material tipo proveniente da serra de Itabirito.

As espécies mais recorrentes na canga estudada foram Periandra mediterranea, Bauhinia rufa, Tibouchina multiflora $(\mathrm{FR}=100 \%)$, Lychnophora pinaster, Baccharis reticularia, Vellozia compacta, Leandra athropurpurea, Stachytarpheta glabra e Acianthera teres. No trabalho de Jacobi et al. (2008), as espécies S. glabra, A. teres e T. multiflora são citadas como comuns no Quadrilátero Ferrífero, apesar da baixa similaridade entre as áreas, ressaltando-se a importância do potencial dessas espécies nos programas de restauração de áreas degradadas por mineração na região.

A família Orchidaceae é de grande importância no ambiente de canga, sendo essa família, em alguns trabalhos como o de Jacobi et al. (2008), uma das mais ricas, com índice de frequência relativa (FR) de 11,06\%. Neste trabalho foram inventariadas nove espécies, sendo A. teres a mais amostrada no ambiente canga $(F A=86,67)$. Segundo Vincent (2004), essa espécie é importante nas áreas de canga e participa da sucessão primária em afloramentos rochosos quartzíticos (ALVES; KOLBEK, 2000; NOGUEIRA et al., 2005 apud JACOBI et al., 2008). A associação com micorrizas é fator imprescindível no ciclo de vida de certas orquídeas epilíticas, sendo alguns fungos simbiontes encontrados mesmo em áreas degradadas pela mineração (NOGUEIRA et al., 2005 apud JACOBI et al., 2008).

Projetos de reabilitação de áreas degradadas devem utilizar uma variedade grande de espécies, e um dos critérios de escolha pode ser a representatividade de suas famílias tanto em número de espécies quanto em número de indivíduos, uma vez que essa é uma indicação do sucesso do táxon num ambiente particular (JACOBI et al., 2008). 
As espécies de reprodução clonal e rizomatosas, como as Poaceae, são espécies importantes na recuperação de áreas degradadas por mineração de ferro, uma vez que contribuem para a estabilização de taludes por crescerem e se espalharem pelo substrato (SHU et al., 2005 apud JACOBI et al., 2008). Segundo Ângelo et al. (2002), a restauração de áreas degradadas feita a partir de semeadura de mistura de sementes de gramíneas com leguminosas e outras espécies herbáceas, arbustivas e arbóreas constitui uma técnica alternativa que poderá trazer resultados satisfatórios. Porém, recentemente, as empresas de mineração começaram a se interessar por espécies nativas nos programas de recuperação (JACOBI et al., 2008).

Ângelo et al. (2002), estudando uma área em reabilitação também no Complexo Alegria, constataram que as espécies nativas Vernonia polyanthes e Leandra sp apresentaram altos índices de regeneração em áreas de campo ferruginoso após a perturbação pela mineração.

Neste estudo, Leandra atropurpurea foi uma espécie com boa amostragem nos ambientes estudados, com IVI $=1,82$, ocupando o $19^{\circ}$ lugar entre as mais importantes na canga couraçada e com IVI $=1,73$ no ambiente rocha, ficando em $22^{\circ}$ lugar entre as mais importantes nesse ambiente.

A recuperação da cobertura vegetal de áreas degradadas é de grande importância à medida que se procura, por meio do uso de espécies adequadas a cada condição ambiental, proteger o solo e, a partir daí, estabelecer condições para a sucessão vegetal, visando atingir uma comunidade mais estável (LIMA, 1986).

Segundo Lima et al. (2006), uma das causas do questionamento da qualidade dos Planos de Recuperação de Áreas Degradadas - PRAD de minas de ferro no Quadrilátero Ferrífero é a ausência de conhecimento prévio sobre as características dos ecossistemas. Portanto, a caracterização das comunidades vegetais de campo ferruginoso em áreas ainda não mineradas constitui importante avanço nos critérios e escolha de espécies vegetais nativas nos programas de reabilitação de áreas degradadas por mineração de ferro no Quadrilátero Ferrífero.

A melhor forma de compreender a estratégia de desenvolvimento de uma comunidade em um ambiente adverso como os campos ferruginosos é analisar como essa comunidade se comportava antes da degradação. Essa análise é feita através do inventário florístico e fitossociológico e da relação dessa comunidade com o tipo de substrato que a suporta, pois assim é possível prever a reação das espécies nos processos de mitigação e recuperação de áreas degradadas.

A lista de espécies apresentada é preliminar se considerar a alta diversidade florística e a dissimilaridade entre os campos ferruginosos do Quadrilátero Ferrífero. Portanto, recomendam-se outros inventários florísticos e fitossociológicos para otimizar o conhecimento sobre esse ecossistema tão ameaçado.

Para os programas de recuperação de áreas degradadas, recomenda-se o aprimoramento do resgate de espécies de campo ferruginoso, bem como estudar como essas plantas se comportam ex situ, a fim de entender melhor o seu mecanismo de adaptação fisiológica in situ, além de constituírem um banco de germoplasma das espécies, principalmente daquelas ameaçadas de extinção.

\section{AGRADECIMENTOS}

À Samarco Mineração S.A., pelo apoio logístico e financeiro; à CAPES, pela bolsa de estudo concedida ao primeiro autor; a Marcos Sobral, pela identificação das Myrtaceae, e Pedro Lage Viana, pela identificação das Poaceae; e à professora Maria Cristina Teixeira Braga Messias, ao Jorge Luiz, pela ajuda nos trabalhos de campo, CNPq 30 3352/2010-8 e Fapemig RDP-00048-10.

\section{REFERÊNCIAS}

ALVES, R. J. V.; KOLBEK, L. Primary sucession on quartzite clifts in Minas Gerais, Brazil.

Biologia Bratislava, v.55, n.1, p.69-83, 2000.

ÂNGELO, J. G. M.; LENA, J. C.; SANTOS, J. B.. Diversidade vegetal em áreas em reabilitação de mineração de ferro, na mina de Alegria, em Mariana, MG. Revista Árvore, v.26, n.2, p.183-192, 2002.

APG. An update of the Angiosperm Phylogeny Group classification for the orders e families of flowering plants: APG II. Botanical Journal of the Linnean Society, v.141 p.399-436, 2003

BRANDÃO, M.; GAVILANES, M. L. Contribuição para o conhecimento da vegetação da Serra da Piedade, MG. In: CONGRESSO NACIONAL DE BOTÂNICA, 35., Manaus, 1984. Anais... Manaus: SBB, 1984. p.34-51. 
BRANDÃO, M. et al. Contribuição para o conhecimento da Cadeia do Espinhaço em Minas Gerais (Serra de Itabirito). - III. Daphne, v.1. n.3, p.39-41, 1991.

BRANDÃO, M.; GAVILANES, M. L.; ARAÚJO, M. G. Aspectos físicos e botânicos de campos rupestres do estado de Minas Gerais. Daphne, v.4, n.1, p.17-38, 1994.

BRANDÃO, M.; FERREIRA, P. B. D.; ARAÚJO, M. G. Mais uma contribuição para o conhecimento da cadeia do Espinhaço em Minas Gerais - VI: Serra do Rola Moça. Daphne, v.7, n.4 p.50-64, 1997.

CLEEF, A.; SILVA, M. F. F. Plant communities of Serra dos Carajás (Pará), Brazil. Boletim do Museu Paranaense Emílio Goeldi, Série Botânica, v.10, p.206-281, 1994.

DORR, J. V. N. Physiographic, stratigraphic and strutural development of the Quadrilátero Ferrífero Minas Gerais, Brazil.. Washington: United States Government, 1969. 110p.

DRUMMOND, G. M. et al. Biodiversidade em Minas Gerais: um atlas para sua conservação. 2.ed. Belo Horizonte: Fundação Biodiversitas, 2005. 222p.

GIULIETTI, A. M. et al. Flora da Serra do Cipó, Minas Gerais: caracterização e lista de espécies.

Boletim Botânico da Universidade de São Paulo, v.9, p.1-151, 1987.

GIULIETTI, A. M.; PIRANI, J. R.; HARLEY, R. M. Espinhaço Range Region - Eastern Brazil. In:

DAVIS, S. D., (Ed.) Centres of plant diversity: a guide and strategy for their conservatoin. TheAmericas. WWF/IUCN Publications Unit., 1997. v.3. p.397-404.

GOODLAND, R.; FERRI, M. G. Ecologia do cerrado. Belo Horizonte: Itatiaia/São Paulo: Universidade de São Paulo, 1979. 193p.

HARLEY, R. M. Flora of the Pico das Almas, Chapada Diamantina-Bahia, Brasil. In: STANNARD, B. L. E. (Ed). The trustees of the Royal Botanic Gardens. Kew: 1995. 853p.
HERZ, N. Metamorphic rocks of the Quadrilátero Ferrífero, Minas Gerais, Brazil. Geological Survey Professionl Paper, v.641, n.1, p.1-81, 1978.

JACOBI C. M.; CARMO F. F.; VINCENT, R. C. Estudo fitossociológico de uma comunidade vegetal sobre canga como subsídio para a reabilitação de áreas mineradas no Quadrilátero Ferrífero, MG. Revista Árvore, v.32, n.2, p.345-353, 2008.

JACOBI, C. M. Afloramentos ferruginosos: um ecossistema diverso e ameaçado. SIMPÓSIO AFLORAMENTOS FERRUGINOSOS NO QUADRILÁTERO FERRÍFERO, 1., 2008, Belo Horizonte. Trabalho completo... Belo Horizonte: Universidade Federal de Minas Gerais, 2008.

JACOBI, C. M.; CARMO, F. F. Diversidade dos campos rupestres ferruginosos no Quadrilátero Ferrífero, MG. Brasil. Megadiversidade, v.4, n.1/2, p.99-116, 2008.

LIMA, W. P. Princípios de hidrologia florestal para o manejo de bacias hidrográficas. São Paulo: Escola Superior de Agricultura Luiz de Queiroz, 1986. 242p.

LIMA, H. M.; FLORES, J. C. C.; COSTA, F. L. Plano de Recuperação de Áreas degradadas versus plano de fechamento de mina: um estudo comparativo. Revista Escola de Minas, v.59, n.4, p.397-402, 2006.

LORENZI, H. Plantas daninhas do Brasil: terrestres, aquáticas parasitas e tóxicas. 3.ed. Nova Odessa: Plantarum, 2000. 608p.

MAGALHÃES, G. M. Sobre os cerrados de Minas Gerais. Anais da Academia Brasileira de Ciências, v.38, (Supl.) p.59-70, 1966.

MOURÃO, A.; STEHMANN, J. R. Levantamento da Flora do Campo Rupestre Sobre Canga Hematítica Couraçada Remanescente na Mina do Brucutu, Barão de Cocais, MG. Rodriguésia, v.58, n.4, p.775-786, 2007.

MUELLER-DOMBOIS, D.; ELLENBERG, G. H. Aims and methods of vegetation ecology. New York: Wiley \& Sons, 1974. 547p. 
NOGUEIRA, R. E. et al.. Fungos micorrízicos associados a orquídeas em campos rupestres na região do Quadrilátero Ferrífero, MG, Brasil. Acta Botânica Brasílica, v.19, n.3, p.417-424. 2005.

PORTO, M. L.; SILVA, M. F. F. Tipos de vegetação mesófila em áreas da Serra de Carajás e de Minas gerais, Brasil. Acta Botânica Brasileira, v.3, n.2, p.13-21, 1989.

RIZZINI, C. T. Tratado de fitogeografia do Brasil; aspectos sociológicos e florísticos. São Paulo: HUCITEC/USP, 1979. 374p

ROSCHEL, M. B. Levantamento florístico fanerogâmico do Campo Rupestre da Estrada da Torre, Antônio Pereira, Ouro Preto, MG. 2000. 133f. Dissertação (Mestrado em Ciências Biológicas) - Universidade Federal do Rio de Janeiro/Museu Nacional, 2000. 133p.

SECCO, R. S.; MESQUITA, A. I. Notas sobre a vegetação de canga da serra Norte - I. Boletim Museu Paranaense Emilio Goeldi, Nova Série Botânica, v.59, p.1-13, 1983.

SILVA. M. F. F.; ROSA, N. A. Estudos botânicos na área do Projeto Ferro Carajás - Serra Norte. I. Aspectos fito-ecológicos dos campos rupestres. In: CONGRESSO NACIONAL DE BOTÂNICA, 35., 1990, Manaus. Anais... Manaus: Sociedade Brasileira de Botânica, 1990. p.367-379.

SILVA. M. F. F. Análise florística da vegetação que cresce sobre Canga Hematítica em Carajás Pará - Brasil. Boletim do Museu

Paranaense Emílio Goeldi, Série de Botânica, v.7, n.1, p.7-22, 1991.

SILVA, M. F. F. Distribuição de metais pesados na vegetação metalófila de Carajás. Acta Botânica, v.6, n.1, p.107-122, 1992.
SILVA, M. F. F.; SECCO, R. S.; LOBO, M. G. A. Aspectos ecológicos da vegetação rupestre da Serra de Carajás, estado do Pará, Brasil. Acta Amazônica, v.26, n.1, p.17-44, 1996.

SHU, W. S. et al.. Natural colonizatoin of plants on five lead/zinc mine tailings in southern China. Restoration Ecology, v.13, n.1, p.49-60, 2005.

TEIXEIRA, W. A.; LEMOS-FILHO, J. P. Metais pesados em folhas de espécies lenhosas colonizadoras de uma área de mineração de ferro em Itabirito, Minas Gerais. Revista Árvore v.22, n.2, p.381-388, 1998.

VIANA, P. L.; LOMBARDI, J. A. Florística e Caracterização dos Campos Rupestres Sobre Canga na Serra da Calçada, Minas Gerais, Brasil. Rodriguésia, v.58, n.1, p.159-177, 2007.

VIANA, P. L. A flora sobre os campos rupestres sobre canga no Quadrilátero Ferrífero. SIMPÓSIO: AFLORAMENTOS FERRUGINOSOS NO QUADRILÁTERO FERRÍFERO, 1., 2008, Belo Horizonte. Trabalho completo. Belo Horizonte: Universidade Federal de Minas Gerais, 2008.

VIANA, P. L.; FILGUEIRAS, T. S. Inventário e distribuição das gramíneas (Poaceae) na Cadeia do Espinhaço, Brasil. Megadiversidade, v.4, n.1-2, p.99-116, 2008.

VINCENT, R. C.; JACOBI, C. M.; ANTONYNY, Y. Diversidade na adversidade. Ciência Hoje, v.31, n.185, p.64-67, 2002.

VINCENT, R. C. Florística, fitossociologia e relações entre a vegetação e o solo em áreas de campos ferruginosos no quadrilátero ferrífero, Minas Gerais. 2004. 144f. Tese (Doutorado em Ecologia) Instituto de Biociências, USP, 2004. 144p. 
